ARTIGO DE PESQUISA - RESEARCH ARTICLE

\title{
TRADITIONAL BOTANICAL KNOWLEDGE VARIATION BETWEEN GENDER AND AGE IN A MAKUXI COMMUNITY IN RORAIMA SAVANNA, NORTHERN BRAZILIAN AMAZONIA
}

\author{
VARIAÇÃO DO CONHECIMENTO BOTÂNICO TRADICIONAL ENTRE GÊNERO E \\ IDADE EM UMA COMUNIDADE MAKUXI EM RORAIMA SAVANNA, AMAZÔNIA DO \\ NORTE DO BRASIL
}

\author{
Rodrigo Leonardo Costa de OLIVEIRA ${ }^{1 *}$, Luis Felipe Paes de Almeida ${ }^{2}$, Veridiana Vizoni SCUDELLER ${ }^{3}$, Reinaldo \\ Imbrozio BARBOSA ${ }^{4}$
}

${ }^{1 *}$ ETNIA, Universidade Estadual de Roraima. rodrigo@uerr.edu.br; ${ }^{2}$ Instituto Insikiran, Universidade Federal de Roraima, ${ }^{3}$ Instituto de Ciências Biológicas, Universidade Federal do Amazonas, ${ }^{4}$ Núcleo de Pesquisas de Roraima, Instituto Nacional de Pesquisas da Amazônia

\begin{abstract}
The studies over the local knowledge patterns between man and woman aim to understand specific characteristics of communities in order to develop better strategies for natural resources conservation. The objective of this study was to recognize the useful species and their forms of use in order to evaluate traditional Makuxi knowledge of genders and age in the Darora community, in São Marcos indigenous land, Roraima State, Northern Brazil. The botanical knowledge was analyzed, considering species richness, number of citations and plant uses between gender and age groups. The research was carried out using semi-structured interviews with 60 participants (36 men and 24 women), ranging from 18 to 84 years of age. The interview data were compared between genders and three age groups $(<40,40-60$, and $>60$ years of age). A total of 69 species and 2.100 citations were recorded (men, 69 species in 1.446 citations; women, 52 species in 654 citations). For the age groups, 53 species were mentioned by the category $<40,61$ by the category $40-60$, and 59 by the category $>60$ years. This difference was also observed in the species uses and in its parts, being timber uses the most significant purposes among men, and medicinal and food among women. The study provided an analysis of the profile of the Darora community, i.e., they are explorers of the natural resources, mainly oriented towards construction, due to the relatively recent occupation of the area (ca. 75 years ago), which evidences that they are at the establishment stage, as well as the need for medicine, probably related to the lack of health care, to the cost of industrial drugs, and also due to the belief on traditional medicine effectiveness. Results show that respondents over 40 years of age have more botanical knowledge than the younger ones. Elder men were considered the most knowledgeable regarding to the number of species and citations.
\end{abstract}

Keywords: ethnobotanical indexes, stem use, woody species, Indigenous community.

\section{RESUMO}

Os estudos sobre os padrões de conhecimento local entre homem e mulher visam compreender as especificidades das comunidades a fim de desenvolver melhores estratégias de conservação dos recursos naturais. O objetivo deste estudo foi reconhecer as espécies úteis e suas formas de uso para avaliar o conhecimento tradicional dos Makuxi sobre gênero e idade na comunidade Darora, na Terra Indígena São Marcos, Estado de Roraima, Norte do Brasil. O conhecimento botânico foi analisado, considerando-se a riqueza de espécies, número de citações e usos das plantas entre os gêneros e faixas etárias. A pesquisa foi realizada por meio de entrevistas semiestruturadas com 60 participantes (36 homens e 24 mulheres), com idades entre 18 e 84 anos. Os dados da entrevista foram comparados entre os gêneros e três faixas etárias (<40, $40-60$ e > 60 anos). Um total de 69 espécies e 2.100 citações foi registrado (homens, 69 espécies em 1.446 citações; mulheres, 52 espécies em 654 citações). Para as faixas etárias, 53 espécies foram citadas pela categoria $<40,61$ pela categoria 40-60 e 59 pela categoria $>60$ anos. Essa diferença também foi observada nos usos das espécies e em suas partes, sendo os usos da madeira as finalidades mais significativas entre os homens, e medicinais e alimentares entre as mulheres. O estudo permitiu uma análise do perfil da comunidade Darora, ou seja, são exploradoras dos recursos naturais, principalmente orientadas para a construção, devido à ocupação relativamente recente da área (há cerca de 75 anos), o que evidencia que eles estão na fase de implantação, bem como a necessidade de medicamentos, provavelmente relacionada à falta de assistência à saúde, ao custo dos medicamentos industriais, e também pela crença na eficácia da medicina tradicional. Os resultados mostram que os entrevistados com mais de 40 anos possuem mais conhecimento botânico do que os mais jovens. Os homens mais velhos foram considerados os mais conhecedores quanto ao número de espécies e citações.

Palavras-chave: índices etnobotânicos, uso do caule, espécies lenhosas, comunidade indígena. 


\section{INTRODUCTION}

Studies on local knowledge patterns have been the focus of ethnobotanical research over the last few years (ALBUQUERQUE et al., 2011). Local knowledge, resulting from several years of environmental relationship and transmitted through generations, includes plants uses in the daily life of each culture, as well as management and conservation practices (CAMOU-GUERRERO et al., 2008; SOP et al. 2012). Such studies have highlighted the organization and distribution of this knowledge under the influence of social factors, such as age, gender, education, occupation, and interaction with different ethnic groups (EYSSARTIER et al., 2008; ALMEIDA et al., 2012; GÓMEZ-BAGGETHUN and REYES-GARCÍA, 2013). Age and gender are the most analyzed factors in ethnobotany studies and provide the local knowledge understanding for implementation actions on resource management, according to the local population's preferences, as specific information is revealed (AYANTUNDE et al., 2008).

One of the trends observed in several ethnobotanical studies is that women have greater knowledge about medicinal species and men about woody species for fuel generation and construction (BOSTER, 1985; LUCENA et al., 2007). This is explained due to labor division between men and women, resulting in different experiences, and consequently different levels of local knowledge (VOEKS, 2007). Moreover, elder individuals have wider knowledge when compared with younger ones, because they are less affected by external factors and by their highest experience (QUINLAN and QUINLAN, 2007). In any case, knowledge loss can be caused by different changes in generations' lifestyle.

In this context, the study aims to bring contributions to the natural resources knowledge of an Amazonian indigenous community in the savanna area, in order to recognize useful species and their respective uses, and to evaluate the variation of traditional botanical knowledge between gender and age.

\section{MATERIAL AND METHODS Study area}

São Marcos Indigenous Land (TISM) comprises 654,110 ha and 42 indigenous communities of the Makuxi, Taurepáng and Wapixana ethnic groups. The study was carried out with a Makuxi ethnic group, from the Darora Community, Lower São Marcos ethno region ( $\left.3^{\circ} 10^{\prime} 42^{\prime \prime} \mathrm{N}, 60^{\circ} 23^{\prime} 34^{\prime \prime} \mathrm{W}\right)$. The study area is located $90 \mathrm{~km}$ from the Roraima State capital, Boa Vista (Figure 1).

Occupation of the Darora Community occurred in the 1940's, when some families from the Xumina Community (Raposa Serra do Sol Indigenous Land) migrated to the region due to the difficulty in finding agricultural areas. The community has strong ability for agriculture and animal livestock, considered as necessary for food security. Extractivism is still practiced, and commonly related to house construction, fences, and fruit collection. There is also a small building where medical and dental care is regularly offered to residents by the government agencies. According to the community's Health Center, there are 40 families living in Darora, totaling 184 people, from 50 of which are men and 43 are women over the age of 18; 5 are men and 6 are women over the age of 60.

There are two public schools in the Darora Community: one for pre-school, supported by the Boa Vista City Hall, and one for elementary and high school, supported by the State of Roraima Government. All residents speak Portuguese fluently. The Makuxi language, from the Karib linguistic trunk, is taught in pre-school; however, the native language is rarely spoken by the residents, with only two teachers and one elder villager as competent speakers.

\section{Ethics and legal aspects}

This study is part of the research project "Use and conservation of plant resources by indigenous communities in Northern Roraima State", of the Graduate Program in Biological Sciences (Botany), of the National Institute of Amazonian Research (INPA) and the Roraima State University (UERR) in Boa Vista. The project was submitted to and approved by the Ethics Committee for Human Research of INPA and the National Commission of Ethics in Research with Humans (CEP-INPA/CONEP) (permit \# 814.370 - 2014). The study was authorized by the National Foundation for Indigenous People (FUNAI) (number 73/AAEP/PRES/2014), and by the National Institute for Historical and Artistic Heritage (IPHAN) (number 15/2014). 


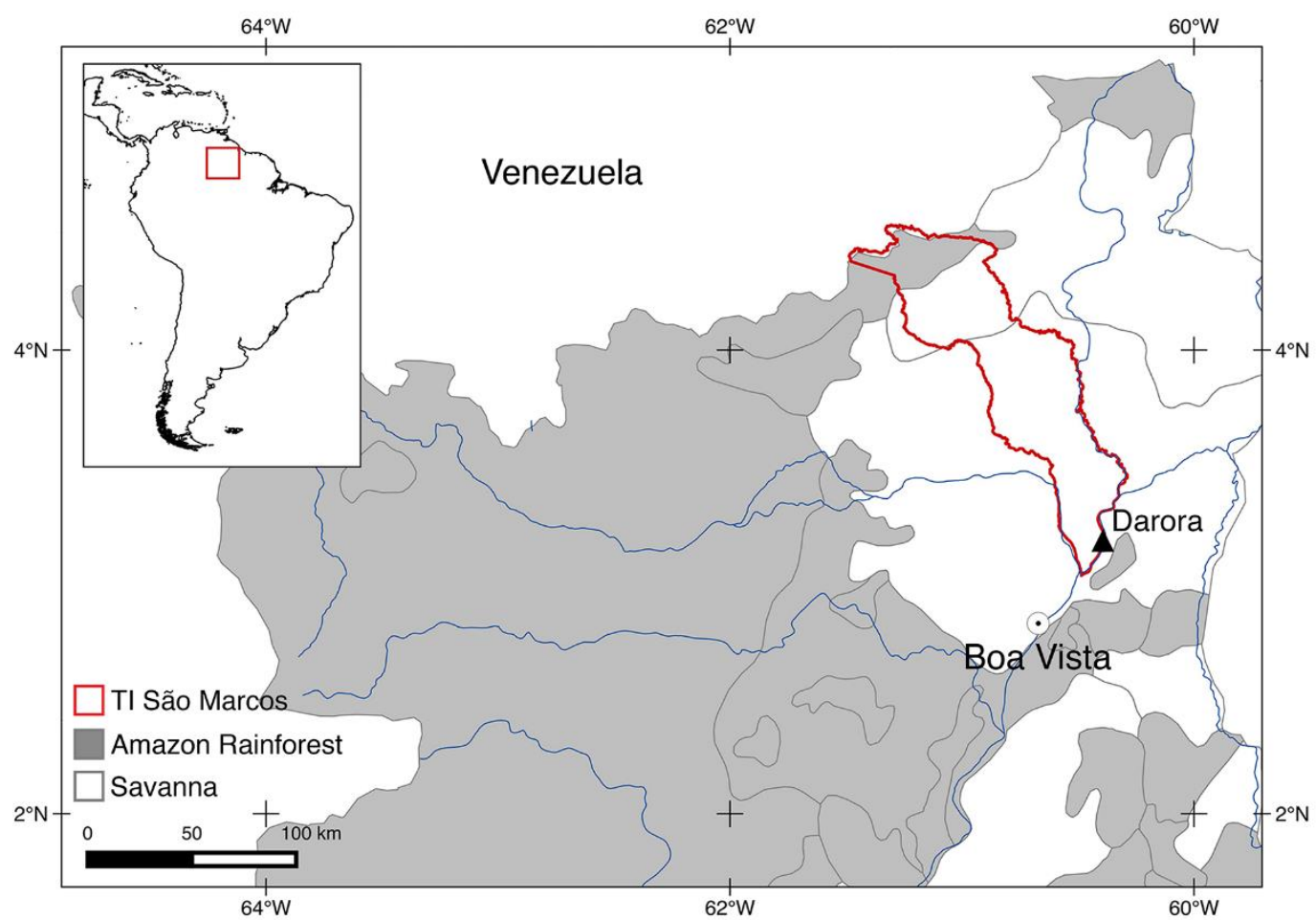

Figure 1. Location of the Darora Indigenous Community - São Marcos Indigenous Land (TISM), Roraima, Brazil.

\section{Ethnobotanical survey}

For ethnobotanical data survey, interviews were carried out with 60 respondents (36 men and 24 women) from 38 families, between 18 and 84 years of age. All the families were visited in the community, and each resident was invited to participate. The purpose of the research was explained, and for those who agreed were given an informed consent term.

Interviews were carried out from November 2014 to November 2015. Firstly, participants were asked where the non-forest (savanna) and forest species were collected, and then the free-list technique was used, i.e., asking for all species they knew, as suggested by Albuquerque et al. (2014). Afterwards, semistructured interviews were carried out (ALBUQUERQUE et al., 2014), with questions about gathering, forms of use, and plants parts used. Responses were grouped into the categories Food, Construction, Medicinal, Fuel, Handicraft, and Technology, adapted and adopted from previous studies (LINS NETO et al., 2008; LUCENA et al., 2012).

\section{Botanical material collection}

Samples were collected and taxonomic identification was carried out by botanists, parabotanists and local flora guides (RIBEIRO et al., 1999; MELO and BARBOSA, 2007; FLORES and RODRIGUES, 2010; WITTMANN et al., 2010; OLIVEIRA et al., 2017). Samples were deposited in the Federal University of Roraima's (UFRR) herbarium in Boa Vista. Some samples were recorded by photographs and others were not collected (Table 1).

\section{Data analysis}

Quantitative analysis was performed considering different measures in the knowledge between genders and ages (BYG and BALSLEV, 2001): Use Diversity Value (UDV) - measures how a species is used within a category and its degree of contribution to its total use; Use of Equability Value (UEV) - measures how the different uses contribute to the total uses of a given species, regardless of the number of use categories; Informant Diversity Value (IDV) - measures how many respondents use a given species and how the species use is distributed among respondents; Informant Equability Value (IEV) - measures how the use of a species is distributed among respondents, regardless of the number of respondents who use the species; Species Diversity Value (SD) - measures how a respondent uses several species and how the different uses are distributed among species; and Species Equability Value (SE) - measures how a respondent uses known plants, regardless of the number of plants used. 
Age groups were defined according to Arévalo-Marín et al. (2015), by dividing the difference between the youngest in age (18 years old) and the oldest in age ( 84 years old) by three, resulting in the number of intervals chosen for this study. Thus, category < 40 years old had 15 men and nine women; category $40-60$ years old had 16 men and 9 women; and category $>60$ years old had five men and six women.

To test cultural variation, groups of men and women were compared, as well as age bracket of members, considering number of species and number of citations. For statistical analysis, the MannWhitney test was performed between the genders, and the Kruskal-Wallis test was carried out between age groups.

\section{RESULTS}

\section{Botanical families and use categories}

Seventy-two plants considered useful were registered, belonging to 26 botanical families. Four species were not identified. Among the botanical families, Fabaceae presented the greatest number of species (21), followed by Arecaceae (6) (Table 1). Within 2.100 citations for species, 1.446 were reported by men (36), comprising 67 plant species (97\% of the total), and 654 were reported by women (24), comprising 52 species (75\% of the total) (Table 1). Eighteen species were mentioned only by men, and two species were mentioned only by women.

Species were included in seven use categories according to the information obtained by the respondents (Table 1 and 2). Among these categories, women were more prominent than men in the Medicinal (50\%), Food (44\%), Fuel (27\%) categories, while men stood out in the Construction (65\%), Technology (47\%), Animal feeding (22\%), and Handicrafts (15\%) categories (Table 2). Percentage value was calculated within each gender group, separately. From the species reported in the study, 11 presented more than one use; for instance, copaiba had 23 different uses, and 59\% of the species had more than three uses. In the construction category, it should be mentioned that different species from forest island and riparian forest environments, such as Mauritia flexuosa, Copaifera pubiflora, Leptolobium nitens and Eschweilera sp. Significant differences $(\mathrm{p}<0.05)$ between genders were observed only in construction and technology categories.

In relation to the age group, 53 species $(77 \%)$ were cited by $<40$ year old respondents, 61 species $(88 \%)$ were cited by category between 40 and 60 years of age, and 59 species $(85 \%)$ were mentioned by the > 60 year old folks. The more cited species by men were Curatella americana (97\%), Copaifera pubiflora, Byrsonima crassifolia and B. coccolobifolia (with 89\%), and the more cited ones by women were Leptolobium nitens (83\%) and Curatella americana (79\%).

Darora community respondents know on average $17( \pm 8)$ useful plant species, and the category < 40-year-old observed members know less species than the other two age groups $(\mathrm{H} \sim 16.137 ; \mathrm{p}<0.05)$, for both genders. Significant differences in the number of species between genders were observed, as men knew a greater number of species in relation to women $(U=222.5 ; \mathrm{p}<0.05)$.

Comparing genders in the category $>40$ years old, visual difference was observed in the knowledge between men and women; however, this difference was statistically insignificant (Table 3 ).

Mean number of citations per respondent was $36( \pm 19)$ for each species. Men had a higher number of citations than women $(\mathrm{U}=257.0, \mathrm{p} \sim 0.008)$. Despite the difference between the number of citations for $<40$ years old respondents, no significant differences were observed between genders. Categories over the age of 40 did not present significant differences among the two sexes; however, older respondents tended to know more about species use in both genders (Table 3).

A further comparison of the species richness between genders, considering each age group, showed categories $40-60$ and > 60 years old were not divergent, which was maintained in the community's total number. Moreover, men know more species than women (Table 3). Considering the number of citations, similar pattern was observed. The group of younger women $(<40)$ indicated lower diversity of citations and is statistically different $(p<0.05)$ from the two groups of older respondents $(40-60)$ and (>60) (Table 2). Men and women presented significant differences in the number of citations between $<40$ and $>60$ years of age, and men cited more species (Table 3 ).

\section{Knowledge $x$ gender $x$ age}

Significant differences $(\mathrm{p}<0.05)$ were observed between the genders regarding the quantitative measures UDV and SD. For respondent diversity value (IDV) knowledge between genders, no differences were observed (Table 4). In relation to the results obtained using the quantitative measures 
IDV and SD, a pattern of age groups 40 - 60 and > 60 was observed, in which these age groups did not present differences between each other over one another and were significantly different from the category $<40$, with $\mathrm{p}<0.05$ (Table 4). UDV was an exception, with no significant differences between the three age groups (Table 3), indicating species have similar quantity of uses in the categories indicated, but the differences occur in the number of respondents and in the diversity of use. In addition, the species knowledge is higher among the elders.

Table 1. Useful woody species in the Darora community, Roraima, Brazil. UFRR - Deposit code of federal University of Roraima Herbarium; cnh - collected but not herborized; pr - photographic record; wc - without collect.

Use categories

\begin{tabular}{|c|c|c|c|c|c|c|c|c|c|}
\hline Family/Species & Common name & نٍ & & $\underset{8}{\mathscr{8}}$ & 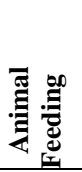 & 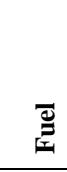 & : & 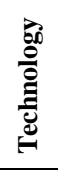 & UFRR \\
\hline \multicolumn{10}{|l|}{ ANACARDIACEAE } \\
\hline $\begin{array}{l}\text { Spondias sp. } \\
\text { ANNONACEAE }\end{array}$ & taperebá & $\mathrm{X}$ & $\mathrm{X}$ & $\mathrm{X}$ & & & $\mathrm{X}$ & $\mathrm{X}$ & $\mathrm{cnh}$ \\
\hline $\begin{array}{l}\text { Guatteria } \text { sp. } \\
\text { Xylopia aromatica (Lam.) Mart. } \\
\text { APOCYNACEAE }\end{array}$ & envireira & $\mathrm{X}$ & & & & & & & $\begin{array}{l}8442 \\
8449\end{array}$ \\
\hline $\begin{array}{l}\text { Himatanthus articulatus (Vahl) Wood. } \\
\text { ARECACEAE }\end{array}$ & sucuba & $\mathrm{X}$ & & $\mathrm{X}$ & & $X$ & $X$ & $\mathrm{X}$ & 8487 \\
\hline Desmoncus sp. & jacitara & & $X$ & & & & & $\mathrm{X}$ & $\mathrm{pr}$ \\
\hline Euterpe precatoria Mart. & acai & $X$ & & $X$ & & & $\mathrm{X}$ & & $\mathrm{pr}$ \\
\hline Mauritia flexuosa L.f. & buriti & $\mathrm{X}$ & $\mathrm{X}$ & $\mathrm{X}$ & $\mathrm{X}$ & $\mathrm{X}$ & & $\mathrm{X}$ & pr \\
\hline BIGNONIACEAE & & & & & & & & & \\
\hline Handroanthus sp. & pau-d'arco & $\mathrm{X}$ & & & & & $\mathrm{X}$ & $\mathrm{X}$ & $\mathrm{cnh}$ \\
\hline $\begin{array}{l}\text { Tabebuia insignis (Miq.) Sandw. } \\
\text { BIXACEAE }\end{array}$ & bacatirana & $\mathrm{X}$ & & & & & & $\mathrm{X}$ & 8637 \\
\hline $\begin{array}{l}\text { Bixa arborea Huber } \\
\text { CACTACEAE }\end{array}$ & urucum-brabo & & $\mathrm{X}$ & & & & & & 8467 \\
\hline $\begin{array}{l}\text { Cereus sp. } \\
\text { CALLOPHYLACEAE }\end{array}$ & mandacaru & & & & & & $\mathrm{X}$ & & pr \\
\hline $\begin{array}{l}\text { Curatella americana } \mathrm{L} \text {. } \\
\text { FABACEAE }\end{array}$ & caimbé & $\mathrm{X}$ & & $\mathrm{X}$ & $\mathrm{X}$ & $\mathrm{X}$ & $\mathrm{X}$ & $\mathrm{X}$ & 8446 \\
\hline Anadenanthera peregrina (L.) Speg. & angico & $\mathrm{X}$ & & $\mathrm{X}$ & & $\mathrm{X}$ & $\mathrm{X}$ & $\mathrm{X}$ & 8475 \\
\hline Andira sp. & manga-braba & $\mathrm{X}$ & & $\mathrm{X}$ & $\mathrm{X}$ & $X$ & $\mathrm{X}$ & $\mathrm{X}$ & 8465 \\
\hline Bauhinia sp. 1 & escada-de-jabuti & & & & & & $\mathrm{X}$ & & wc \\
\hline Bauhinia sp. 2 & mororó & $\mathrm{X}$ & & $\mathrm{X}$ & & & $\mathrm{X}$ & $\mathrm{X}$ & 8688 \\
\hline Bowdichia virgilioides Kunth. & paricarana & $\mathrm{X}$ & & $\mathrm{X}$ & & $\mathrm{X}$ & $\mathrm{X}$ & $\mathrm{X}$ & 8471 \\
\hline Cassia moschata Kunth. & mari-mari & $\mathrm{X}$ & $\mathrm{X}$ & $\mathrm{X}$ & $\mathrm{X}$ & $\mathrm{X}$ & $\mathrm{X}$ & $\mathrm{X}$ & 8452 \\
\hline Cassia sp. & jurema & $\mathrm{X}$ & & $\mathrm{X}$ & & $\mathrm{X}$ & & & 8456 \\
\hline Copaifera pubiflora Benth. & copaiba & $\mathrm{X}$ & & $\mathrm{X}$ & $\mathrm{X}$ & $\mathrm{X}$ & $\mathrm{X}$ & $\mathrm{X}$ & 8454 \\
\hline Cynometra bauhiniifolia Benth. & copaibinha & $\mathrm{X}$ & & & & & & & 8460 \\
\hline Etabalia dubia Rudd. & mututi & $\mathrm{X}$ & & & & $\mathrm{X}$ & & & 8459 \\
\hline Fabaceae sp. 1 & jauari & $\mathrm{X}$ & & $\mathrm{X}$ & $\mathrm{X}$ & & & & wc \\
\hline Fabaceae sp. 2 & coração-de-nego & $\mathrm{X}$ & & & & & & & 8681 \\
\hline Fabaceae sp. 3 & unha-de-gato & & & & & & $\mathrm{X}$ & & 8670 \\
\hline $\begin{array}{l}\text { Hydrochorea corymbosa (Rich.) Barneby \& } \\
\text { J.W.Grimes }\end{array}$ & vassourinha & $\mathrm{X}$ & & & & $\mathrm{X}$ & & $\mathrm{X}$ & 8468 \\
\hline Inga sp. 1 & inga & & & $\mathrm{X}$ & & & & & 8677 \\
\hline Inga sp. 2 & ingarana & $\mathrm{X}$ & & $\mathrm{X}$ & $\mathrm{X}$ & $X$ & & $\mathrm{X}$ & 8704 \\
\hline
\end{tabular}




\begin{tabular}{|c|c|c|c|c|c|c|c|c|c|}
\hline \multirow[b]{2}{*}{ Family/Species } & \multirow[b]{2}{*}{ Common name } & \multicolumn{7}{|c|}{ Use categories } & \multirow[b]{2}{*}{ UFRR } \\
\hline & & Uี & : & $\ddot{\circ}$ & 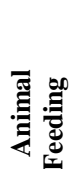 & 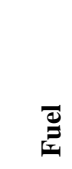 & 㺼 & 常 & \\
\hline Leptolobium nitens Vogel & darora & $\mathrm{X}$ & $\mathrm{X}$ & $\mathrm{X}$ & & $\mathrm{X}$ & $\mathrm{X}$ & & $\mathrm{cnh}$ \\
\hline Machaerium acacifolium Benth. & arapari & $\mathrm{X}$ & $\mathrm{X}$ & $\mathrm{X}$ & & $\mathrm{X}$ & & $\mathrm{X}$ & 8697 \\
\hline Ormosia smithii Rudd & tento & & $\mathrm{X}$ & $\mathrm{X}$ & & $\mathrm{X}$ & & & 8437 \\
\hline Peltogyne floribunda (Kunth) Pittier & roxinho & $\mathrm{X}$ & & & & & & & 8660 \\
\hline $\begin{array}{l}\text { Swartzia sp. } \\
\text { HYPERICACEAE }\end{array}$ & piritó & $\mathrm{X}$ & & & $\mathrm{X}$ & $\mathrm{X}$ & & & 8478 \\
\hline $\begin{array}{l}\text { Vismia cayennensis Aubl. } \\
\text { HUMIRIACEAE }\end{array}$ & lacre & $\mathrm{X}$ & & $\mathrm{X}$ & & & $\mathrm{X}$ & & 8670 \\
\hline $\begin{array}{l}\text { Humiria balsamifera Aubl. } \\
\text { LAURACEAE }\end{array}$ & miri & $\mathrm{X}$ & & $\mathrm{X}$ & & & & $\mathrm{X}$ & 8429 \\
\hline $\begin{array}{l}\text { Endlicheria sp. } \\
\text { LECYTHIDACEAE }\end{array}$ & louro & $\mathrm{x}$ & & & & & & & 8432 \\
\hline Couratari multiflora (J. Smith) Eyma & tauari & & & & & & & $\mathrm{X}$ & 8659 \\
\hline $\begin{array}{l}\text { Eschweilera } \text { sp. } \\
\text { LOGANIACEAE }\end{array}$ & macaco-aricuia & $\mathrm{X}$ & & $\mathrm{X}$ & & & & $\mathrm{X}$ & 8463 \\
\hline $\begin{array}{l}\text { Antonia ovata Pohl. } \\
\text { MALPIGHIACEAE }\end{array}$ & timbó & & & & & & & $\mathrm{X}$ & 8485 \\
\hline $\begin{array}{l}\text { Byrsonima crassifolia }(\mathrm{L} \text {.) Kunth. } \\
\text { Byrsonima coccolobifolia Kunth. }\end{array}$ & mirixi & & $\mathrm{X}$ & $\mathrm{X}$ & & $\mathrm{X}$ & $\mathrm{X}$ & & $\begin{array}{l}8482 \\
8483\end{array}$ \\
\hline Byrsonima sp. & mirixi-de-galega & $\mathrm{X}$ & & $\mathrm{X}$ & & $\mathrm{X}$ & $\mathrm{X}$ & & 8443 \\
\hline $\begin{array}{l}\text { Byrsonima verbascifolia (L.) DC. } \\
\text { MALVACEAE }\end{array}$ & orelha-de-burro & & & $\mathrm{X}$ & & & $\mathrm{X}$ & & 8480 \\
\hline $\begin{array}{l}\text { Ceiba sp. } \\
\text { MYRISTICACEAE }\end{array}$ & samaúma & $\mathrm{X}$ & & & & & & $\mathrm{X}$ & wc \\
\hline $\begin{array}{l}\text { Virola molissima Poepp. ex. A. DC. } \\
\text { Virola surinamensis (Rol. ex Rottb.) Warb. } \\
\text { MYRTACEAE }\end{array}$ & ucuuba & $\mathrm{X}$ & $\mathrm{X}$ & & & & $\mathrm{X}$ & $\mathrm{X}$ & $\begin{array}{l}8430 \\
8635\end{array}$ \\
\hline Eugenia punicifolia (Kunth) DC. & pimenta-de-raposa & & & $\mathrm{X}$ & & & $\mathrm{X}$ & & 8682 \\
\hline $\begin{array}{l}\text { Eugenia sp. } \\
\text { Myrcia sp. }\end{array}$ & goiabinha & $\mathrm{X}$ & & $\mathrm{X}$ & $\mathrm{X}$ & & & & $\begin{array}{l}8464 \\
8453\end{array}$ \\
\hline Myrciaria dubia (Kunth) McVaughn. & caçari & & & $\mathrm{X}$ & & & $\mathrm{X}$ & & 8698 \\
\hline Psidium sp. 1 & araçá-do-lavrado & $\mathrm{X}$ & & $\mathrm{X}$ & $\mathrm{X}$ & $\mathrm{X}$ & $\mathrm{X}$ & & 8689 \\
\hline Psidium sp. 2 & $\begin{array}{l}\text { araçá-da-beira-do- } \\
\text { rio }\end{array}$ & & & $\mathrm{X}$ & $\mathrm{X}$ & & $\mathrm{X}$ & & wc \\
\hline $\begin{array}{l}\text { OPILIACEAE } \\
\text { Agonandra silvatica Miers ex Benth. \& } \\
\text { Hook.f. } \\
\text { POLYGONACEAE }\end{array}$ & marfim & & $\mathrm{X}$ & $\mathrm{X}$ & $\mathrm{X}$ & & & & 8477 \\
\hline $\begin{array}{l}\text { Coccoloba } \text { sp. } \\
\text { PROTEACEAE }\end{array}$ & caraoacu & $\mathrm{X}$ & & & & & & & 8694 \\
\hline $\begin{array}{l}\text { Roupala montana Aubl. } \\
\text { RUBIACEAE }\end{array}$ & congonha & & & $\mathrm{X}$ & & $\mathrm{X}$ & $\mathrm{X}$ & & 8484 \\
\hline Genipa americana $\mathrm{L}$. & jenipapo & $\mathrm{X}$ & & $\mathrm{X}$ & $\mathrm{X}$ & $\mathrm{X}$ & $\mathrm{X}$ & $\mathrm{X}$ & 8488 \\
\hline Genipa duckei Steyerm. & jenipapo-brabo & & & $\mathrm{X}$ & $\mathrm{X}$ & & $\mathrm{X}$ & $\mathrm{X}$ & 8458 \\
\hline $\begin{array}{l}\text { Palicourea rigida Kunth. } \\
\text { SALICACEAE }\end{array}$ & douradão & & & $\mathrm{X}$ & & & $\mathrm{X}$ & & 8489 \\
\hline $\begin{array}{l}\text { Casearia sylvestris } \mathrm{Sw} . \\
\text { URTICACEAE }\end{array}$ & canela-de-veio & & & & & & $\mathrm{X}$ & $\mathrm{X}$ & 8631 \\
\hline $\begin{array}{l}\text { Cecropia sp. } \\
\text { VERBENACEAE }\end{array}$ & embaúba & & $\mathrm{X}$ & $\mathrm{X}$ & & & $\mathrm{X}$ & $\mathrm{X}$ & 8680 \\
\hline $\begin{array}{l}\text { Vitex schomburgkiana Schauer } \\
\text { INDETERMINATE }\end{array}$ & maria-preta & $\mathrm{x}$ & & $\mathrm{X}$ & $\mathrm{X}$ & $\mathrm{X}$ & & $\mathrm{X}$ & 8470 \\
\hline Indet 1 & abiorana & $\mathrm{X}$ & & $\mathrm{X}$ & $\mathrm{X}$ & & & & 8700 \\
\hline Indet 2 & aroeira & $\mathrm{X}$ & & & & & $\mathrm{X}$ & & 8664 \\
\hline Indet 3 & marupá & $\mathrm{X}$ & $\mathrm{X}$ & $\mathrm{X}$ & $\mathrm{X}$ & & & $\mathrm{X}$ & wc \\
\hline Indet 4 & pau-carlito & $\mathrm{X}$ & & & & & & $\mathrm{X}$ & 8699 \\
\hline
\end{tabular}




\section{Parts of the plant used by the respondents}

Stems had the highest total number of citations (1.051) and the highest percentage among men in relation to women, followed by flowers/fruit/seed (451) and bark (344). Women presented higher citation percentage than men for flowers/fruit/seed, bark, leaf and oil/latex. In addition to the stem uses, men also had higher citation percentage for root uses (Table 5). Stem was cited in all six use categories, followed by leaf (five) and flower/fruit/seed (four).

Table 2. Number and percentage of species mentioned by women and men in each use category. Numbers followed by the same letter presented no significant differences by the Mann-Whitney test.

\begin{tabular}{|c|c|c|c|}
\hline \multirow[t]{2}{*}{ Use category } & \multirow[t]{2}{*}{ Types of use } & \multicolumn{2}{|c|}{$\begin{array}{l}\text { Mentioned species/ } \\
(\%)\end{array}$} \\
\hline & & Women & Men \\
\hline Construction & $\begin{array}{l}\text { Wood for stakes, fence, pole, rafter, wooden slat, wall, crossbeam, } \\
\text { door, window, roof structure. House cover for pigs and hens. }\end{array}$ & $\begin{array}{c}26^{\mathrm{a}} \\
(50 \%)\end{array}$ & $\begin{array}{c}44^{\mathrm{b}} \\
(65 \%)\end{array}$ \\
\hline Handicraft & $\begin{array}{l}\text { Fiber to make dance garments; woodwork; seed for necklaces, } \\
\text { beads, bracelets. Fruit and Seed for body painting or for buriti fiber } \\
\text { dyeing. }\end{array}$ & $7^{\mathrm{a}}$ & $\begin{array}{c}10^{\mathrm{a}} \\
(15 \%)\end{array}$ \\
\hline Food & $\begin{array}{l}\text { Fruit for in natura consumption, juice, popsicle, pulp, jam or sweet } \\
\text { preserves. Leaf for tea. Palm heart. }\end{array}$ & $\begin{array}{c}23^{\mathrm{a}} \\
(44 \%)\end{array}$ & $\begin{array}{c}29^{b} \\
(43 \%)\end{array}$ \\
\hline Animal Feeding & Fruit and seed for animal feeding & $\begin{array}{c}7^{\mathrm{a}} \\
(13 \%)\end{array}$ & $\begin{array}{c}15^{\mathrm{b}} \\
(22 \%)\end{array}$ \\
\hline Fuel & Core stem for firewood and charcoal. & $\begin{array}{c}14^{\mathrm{a}} \\
(27 \%)\end{array}$ & $\begin{array}{c}17^{\mathrm{a}} \\
(25 \%)\end{array}$ \\
\hline Medicinal & $\begin{array}{l}\text { Bark and inner bark are used for tea; soaking; bathing; syrup; and } \\
\text { medicinal bottle preparation (garrafada); anti-inflammatory; vaginal } \\
\text { bath; child's bath; malaria; intestinal worm; cough; dysentery; } \\
\text { stomachache; diarrhea; itching; wound washing; pain in general; } \\
\text { liver, kidney inflammation; skin diseases; gargling; animal } \\
\text { dysentery; ulcer, gastritis, diabetes; bone, muscles and spine pain; } \\
\text { fever; flu; jaundice; nausea; high blood pressure; urinary infection; } \\
\text { high cholesterol; uterus inflammation; menstruation regulation; } \\
\text { cancer. Leaf is used in infusion; macerated; in tea and syrup as } \\
\text { healing; anti-inflammatory; wounds washing; against jaundice and } \\
\text { anemia; for high cholesterol and high blood pressure; diarrhea; } \\
\text { dysentery; pain in general; urinary infection; fever; for bladder and } \\
\text { kidney stone. Fruit is used in juice, syrup or sauce against anemia and } \\
\text { diabetes. Latex and oil are used against gastritis, worms, } \\
\text { inflammation in general, uterine inflammation, gastritis, pain in } \\
\text { general, pneumonia, cancer, dysentery, wounds washing. Root is used } \\
\text { in tea, macerated, in soaking for hemorrhoids, worm, tummy ache, } \\
\text { dysentery, malaria and menstrual cramps. }\end{array}$ & $\begin{array}{c}26^{\mathrm{a}} \\
(50 \%)\end{array}$ & $\begin{array}{c}26^{\mathrm{a}} \\
(39 \%)\end{array}$ \\
\hline Technology & $\begin{array}{l}\text { Wood for bow, arrow, canoe, boat, oar, motor bush, spit, stick, } \\
\text { furniture in general, cabinet, chair, clipboard, stool, table, bench, hoe, } \\
\text { shovel, and sickle handle, musical instrument, saddle, fish trap, pestle, } \\
\text { leather stretching. Stem for green manure. Fiber for sieve, tipiti, } \\
\text { jamaxi. Leaf to wrap food, for ink, fertilizer, to clean pots and dishes, } \\
\text { to kill fish. Latex for paper pasting. Fruit is used as bait, to make } \\
\text { smoke to avoid maruim (a type of mosquito). }\end{array}$ & $\begin{array}{c}18^{\mathrm{a}} \\
(3 \%)\end{array}$ & $\begin{array}{c}32^{\mathrm{b}} \\
(47 \%)\end{array}$ \\
\hline
\end{tabular}


Table 3. Knowledge distribution of the woody species use by gender and age groups. Columns followed by the same letter indicate no significant differences by the Kruskal-Wallis test. *Mean differences using the MannWhitney test. X - mean; SD - standard deviation.

\begin{tabular}{|c|c|c|c|c|c|c|c|c|}
\hline \multirow{3}{*}{$\begin{array}{l}\text { Age } \\
\text { group }\end{array}$} & \multirow{2}{*}{\multicolumn{2}{|c|}{$\begin{array}{l}\text { Number of } \\
\text { respondents }\end{array}$}} & \multicolumn{3}{|c|}{ Mean number of species } & \multicolumn{3}{|c|}{ Mean number of citations } \\
\hline & & & \multirow{2}{*}{$\begin{array}{l}\text { Women } \\
\mathrm{X} \pm \mathrm{SD}\end{array}$} & \multirow{2}{*}{$\begin{array}{c}\text { Men } \\
\text { X } \pm \text { SD }\end{array}$} & \multirow{2}{*}{$\begin{array}{l}\text { General } \\
\mathrm{X} \pm \mathrm{SD}\end{array}$} & \multirow{2}{*}{$\begin{array}{l}\text { Women } \\
X \pm S D\end{array}$} & \multirow{2}{*}{$\begin{array}{c}\text { Men } \\
\mathbf{X} \pm \text { SD }\end{array}$} & \multirow{2}{*}{$\begin{array}{l}\text { General } \\
\mathrm{X} \pm \mathrm{SD}\end{array}$} \\
\hline & Women & Men & & & & & & \\
\hline$<40$ & 9 & 15 & $9.89 \pm 4.99^{\mathrm{a}}$ & $14.27 \pm 7.87^{\mathrm{a}}$ & $12.63 \pm 7.14^{\mathrm{a}}$ & $18.5 \pm 13.1^{\mathrm{a}}$ & $27.9 \pm 16.8^{\mathrm{a}}$ & $24.4 \pm 15.9^{a}$ \\
\hline $40-60$ & 9 & 16 & $15.56 \pm 4.28^{\mathrm{b}^{*}}$ & $23.44 \pm 7.26^{\mathrm{b}^{*}}$ & $20.60 \pm 7.34^{\mathrm{b}}$ & $34.8 \pm 9.7^{b^{*}}$ & $51.5 \pm 18.8^{\mathrm{b}^{*}}$ & $45.5 \pm 17.8^{b}$ \\
\hline$>60$ & 6 & 5 & $15.33 \pm 4.93^{\mathrm{b}^{*}}$ & $26.20 \pm 5.26^{\mathrm{b}^{*}}$ & $20.27 \pm 7.44^{\mathrm{b}}$ & $32.6 \pm 10.6^{\mathrm{b}^{*}}$ & $53.6 \pm 15.4^{\mathrm{b}^{*}}$ & $42.1 \pm 16.4^{b}$ \\
\hline Total & 24 & 36 & $13.38 \pm 5.28^{*}$ & $20.00 \pm 8.69^{*}$ & $17.35 \pm 8.15$ & $28.2 \pm 13.3^{*}$ & $42 \pm 20.9^{*}$ & $36.4 \pm 19.3$ \\
\hline
\end{tabular}

Table 4. Quantitative knowledge indexes calculated for useful species by gender and age groups of the community Darora, Roraima, Brazil. Columns followed by the same letters indicate no significant differences by the KruskalWallis test (age), and * indicate differences using the Mann-Whitney test. (genders). X - mean; SD - standard deviation and minimum and maximum values (in parentheses). Use diversity value (UDV); Use equability value (UEV); Informant diversity value (IDV); Informant equability value (IEV); Species diversity value (SD); Species equability value (SE).

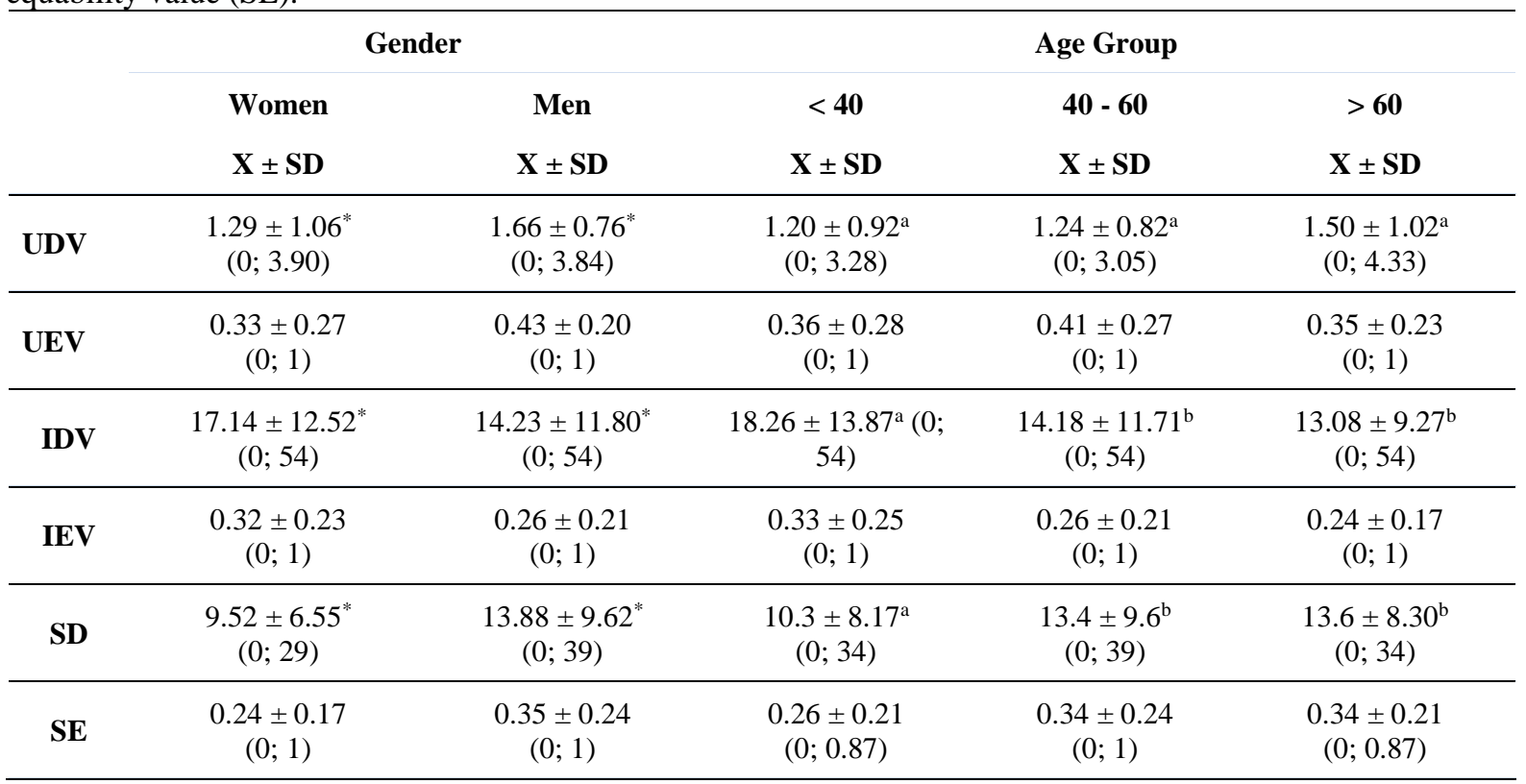

Table 5. Useful parts of plants followed by use categories and number of citations by women and men in the Darora Community.

\begin{tabular}{llllll}
\hline \multirow{2}{*}{ Part of the plant } & Use category & \multicolumn{2}{c}{ Number of citations } & \multicolumn{2}{c}{ Percentage } \\
\cline { 3 - 5 } & Women & Men & Women & Men \\
\hline \multirow{2}{*}{ Bark } & Handicraft & - & 1 & & \\
& Medicinal & 154 & 170 & 28.6 & 24.0 \\
& Technology & 3 & 16 & \\
Flower/Fruit/Seed & Food & 128 & 238 & \\
& Handicraft & 9 & 8 & 20.3 \\
& Medicinal & 10 & 4 & \\
& Animal Feeding & 9 & 41 & \\
\hline
\end{tabular}




\begin{tabular}{|c|c|c|c|c|c|}
\hline \multirow{2}{*}{ Part of the plant } & \multirow{2}{*}{ Use category } & \multicolumn{2}{|c|}{ Number of citations } & \multicolumn{2}{|c|}{ Percentage } \\
\hline & & Women & Men & Women & Men \\
\hline \multirow{5}{*}{ Leaf } & Food & 1 & - & \multirow{5}{*}{11.6} & \multirow{5}{*}{7.2} \\
\hline & Handicraft & 13 & 16 & & \\
\hline & Construction & 15 & 26 & & \\
\hline & Medicinal & 36 & 52 & & \\
\hline & Technology & 11 & 10 & & \\
\hline Oil/Latex & Medicinal & 21 & 32 & 3.2 & 2.2 \\
\hline Root & Medicinal & - & 21 & - & 1.5 \\
\hline \multirow{6}{*}{ Stem } & Food & - & 2 & \multirow{6}{*}{37.2} & \multirow{6}{*}{55.9} \\
\hline & Handicraft & 2 & 7 & & \\
\hline & Fuel & 44 & 74 & & \\
\hline & Construction & 163 & 601 & & \\
\hline & Medicinal & 3 & - & & \\
\hline & Technology & 31 & 124 & & \\
\hline
\end{tabular}

\section{DISCUSSION}

The categories of use, Construction, Medicinal and Food citations were the most divergent between genders, being Construction the most representative among men, and Medicinal the most representative among women. The categories Fuel and Handicraft had low values. However, fuel is one of the most cited in the use of firewood in studies carried out in other environments, such as Brazilian Cerrado (TUNHOLI et al., 2013) and Caatinga (FERRAZ et al., 2006; LUCENA et al., 2007). Handicraft is among the main use categories in the Amazon region (ALARCÓN and PEIXOTO, 2008). The use of species as timber is among the most important use category in Amazonian populations, and has shown to be a very common practice, according to studies carried out in the region (GALEANO, 2000; ALARCÓN and PEIXOTO, 2008; DEMARCHI, 2014).

This practice, when associated with the results of the number of citations of the other categories of timber use, could indicate great local impact on these species (LUOGA et al., 2000). In this study, in agreement with Begossi et al. (2002), Taita (2003), Camou Guerrero et al. (2008), and Tunholi et al. (2013), the use patterns for men and women showed that men have greater knowledge of wood products, and women have greater interest related to the use of food and medicinal plants. In addition, this study provided a profile analysis of the Darora Community residents, as they are natural resources gatherers. The main use is for house construction materials, and afterwards food and medicinal plants. This is explained by the relatively recent settlement in the area, approximately 75 years ago.

On the other hand, comparative analysis of the number of species and citations indicated great differences between genders, being suggesting that men were more knowledgeable than women, and between age groups, being older categories more knowledgeable than younger ones, evidencing confirming patterns found in other studies (ARÉVALO-MARÍN et al., 2015; ALMEIDA et al., 2012; LADIO and LOZADA, 2009). In these studies, such differences are understood as the accumulation of knowledge through previous the experiences of the older generations and their greater interaction with plant resources of the environment. Thus, different ages may have different levels and types of knowledge regarding the use of plants (PHILLIPS and GENTRY, 1993). Such behavior is easily perceived and justified in the Darora community, as the contact of the youngest residents with a nearby urban center, the capital city of Boa Vista, has increased the chances for jobs and continued education, causing traditional knowledge devaluation and a possible transmission rupture of local botanical knowledge.

Greater knowledge among men when compared to women, and the better distribution of knowledge about species among use categories may be related to their position in the community: the man is the hunter and explorer of natural resources, the one who explores the environment outside of the household, 
restricted mainly to woody species; and women's activities are related to family sustenance, such as food, fuel and medicinal care, as also observed by Lucena et al. (2007) and Voeks (2007).

The diversity factor of the species can also contribute to the difference between genders. The Darora community, although located in a savanna region, is surrounded by different phytophysiognomies, such as the riparian forest, "buritizais" and forest islands, which present diverse woody species and a greater variety of the non-forest savanna environment.

On the other hand, Souto and Ticktin (2012), in a community in Venezuela, observed that the effect of gender on knowledge depends on age, where the older woman was more knowledgeable than the younger one. In relation to men, the differences in the level of knowledge were not so variable noticeable. Thus, the knowledge gap between young women and men disappears as they age. To some extent, this characteristic is observed in the Darora Community, since the groups over the age of 40 showed differences only for the younger residents. However, due to the new sociocultural factors that influence the community, younger residents may not reach the same knowledge level as their older folk.

The same pattern was also reported in a savanna environment in southwestern Niger by Ayantunde et al. (2008), where the authors observed a curvilinear pattern between the number of species identified and the age of the respondents, suggesting that knowledge increases from a certain age, and it accumulates over time. This pattern (older residents are more knowledgeable than younger ones) was also observed by Viu et al. (2010) in the Brazilian Cerrado, and by Sop et al. (2012) in the semi-arid vegetation of Burkina Faso. Such behavior was also reported by Albuquerque et al. (2011) in a study with the Fulni-Ô Indians in northeastern Brazil. The authors also found that knowledge declines over older age. This trend was also observed by Almeida et al. (2012) in the Atlantic Forest in Northeastern Brazil, where respondents over the age of 60 presented reduced recall of species richness. Conversely, Lykke et al. (2004) found that age did not influence local knowledge in the Fulani villages in Burkina Faso.

However, studies have not confirmed that differences in knowledge among age groups represent an erosion of knowledge. As also observed by Voeks and Leony (2004), younger residents are still being exposed to the culture and are still forming their knowledge about the local species. This means that the dynamism in the use of the resource may have modified the availability of the species, and younger respondents showed memory of only those species that existed at that time of the interview. This can be confirmed by the study of Fentahun and Hager (2009) in Ethiopia, where respondents under the age of 30 had greater knowledge about wild fruits than older respondents as a result of the greater contact of this generation with natural vegetation. However, sociocultural factors may also interfere with the distribution of local knowledge among age groups (SOP et al., 2012). This fact can be investigated in the Darora community, since indigenous people who provide services to community, such as health agents, general services assistants, and teachers tend to know fewer species than those who work directly in the field.

\section{Parts of the plant - overexploitation of resources}

Stem bark stands out as the most widely used resource, which is similar to studies performed in the semi-arid environment of the Caatinga vegetation in Northeastern Brazil (LUCENA et al., 2007). This is because stem bark is a resource available during the whole year, contrary to leaves and reproductive structures, which are transitory in nature, falling during the dry season. In this way, the continuous use of the stem for different activities could influence the structure of more preferred species by the population, causing local extinction. This is confirmed by Cheikhyoussef et al. (2011), who found the root to be the most used part of the plant, followed by the leaves and the whole plant by different ethnic groups in a savanna environment in Namibia.

In the present study, the great number of citations of stem use is closely linked to the Construction category. This use may have been evidenced by recent practice in the construction of a community housing, a Catholic church, and the implementation of electric power poles in the houses in the vicinity of the central village of the community. These activities involved the participation of men and women, not only in the preparation of food, but also in the preparation of paxiúba, which is the wood slat produced from the Euterpe precatoria or Mauritia flexuosa stem. 
The use of flowers/fruits/seeds and stem bark are linked to the Food and Medicinal categories, respectively, which requires attention to these extractive activities of less impact and monitoring of the species used. Such activities may characterize the community's young age, since residents between the age of 20 and 40 correspond to the third and fourth generations, once the community was founded in 1941. Thus, the construction characteristics meet the new demands, such as population growth and resources advancement, such as electricity. No management practices were observed for woody species. Ocasionally, some species of the forest environments were found in the backyards of the residences, such as Copaifera pubiflora, due to its medicinal use and to the shade they provide. Exotic mango (Mangifera indica) is the preferred tree for shade, since the community is located in the non-forested area of the savanna known as lavrado in the region.

\section{CONCLUSION}

Results suggest that indigenous people of the savanna display substantial knowledge on the native wood species. However, it was possible to identify which age and gender can influence community knowledge, since the respondents over the age of 40 showed greater knowledge than younger respondents, and the older men were considered as the most knowledgeable in relation to the number of species and citations.

The difference in knowledge between men and women is also verified in relation to the purposes and parts of the plants used. The categories Construction and Technology, which were reported as of greater interest to the men, presented the stem as the main useful part of the plant. Conversely, reproductive structures, leaves and bark were reported to be of greater interest to women.

The use of natural resources by the community seems to occur as a result of labor and social organization in relation to gender, which can be confirmed in floristic studies correlated to the local botanical knowledge. These studies may clarify possible gaps for the management and conservation of species, since the elderly may be reporting species that are no longer so abundant and important in the daily life of the community. This fact evidences that, in the past, there might have been greater demand for goods than the environment's capacity.

\section{REFERENCES}

ALARCÓN, J.G.S.; PEIXOTO, A.L. Use of terra firme Forest by Caicubi Caboclos, Middle Rio Negro, Amazonas, Brazil. A quantitative study. Economic Botany 62(1): 60-73, 2008.

ALBUQUERQUE, U.P. et al. Selection of research participants. In: ALBUQUERQUE, U.P.; CUNHA, L.V.F.C.; LUCENA, R.F.P.; ALVES, R.R.N. (eds.). Methods and techniques in ethnobiology and ethnoecology. New York: Springer Protocols Handbooks, 2014. p. 1-13.

ALBUQUERQUE, U.P. et al. The use of plants in the medical system of the Fulni-ô people (NE Brazil): A perspective on age and gender. Journal of Ethnopharmacology, 133: 866-873, 2011.

ALMEIDA, C.F.C.B.R. et al. Intracultural variation in the knowledge of medicinal plants in an urban-rural community in the Atlantic forest from Northeastern Brazil. Evidence-based Complementary and Alternative Medicine, 1-15. 2012.

ANGIOSPERM PHYLOGENY GROUP IV. An update of the Angiosperm Phylogeny Group classification for orders and families of flowering plants: APG IV. Botanical Journal of the Linnean Society, 181: 1-20, 2016.

ARÉVALO-MARÍN, E. et al. Traditional knowledge in a rural community in the semi-arid region of Brazil: age and gender patterns and their implications for plant conservation. Ethnobotany research and applications, 14: 331-344, 2015.

AYANTUNDE, A.A. et al. Botanical knowledge and differentiation by age, gender and ethnicity in Southwestern Niger. Human Ecology, 36: 881-889, 2008.

BARBOSA, R.I. Distribuição das chuvas em Roraima. In: BARBOSA, R.I.; FERREIRA, E.J.; CASTELLON, E.G. (eds.). Homem, ambiente e ecologia no Estado de Roraima. Manaus: Instituto Nacional de Pesquisas da Amazônia, 1997. p. 325-335.

BEGOSSI, A. et al. Medicinal plants in the Atlantic forest (Brazil): knowledge, use, and conservation. Human Ecology, 30(3): 281-299, 2002.

BOSTER, J. S. Requiem for the omniscient informant: there's life in the old girl yet. In: DOUGHERTY J. (ed.). Directions in cognitive anthropology. Illinois: University of Illinois Press, 1985. p. 177-197.

BYG, A.; BALSLEV, H. Diversity and use of palms in Zahamena, eastern Madagascar. Biodiversity and Conservation, 10: 951-970, 2001.

CAMOU-GUERRERO, A. et al. Knowledge and use value of plant species in a Rarámuri community: A gender perspective for conservation. Human Ecology, 36: 259-272, 2008. 
CHEIKHYOUSSEF, A. et al. Ethnobotanical study of indigenous knowledge on medicinal plant use by traditional healers in Oshikoto Region, Namibia. Journal of Ethnobiology and Ethnomedicine, 7, 10. 2011.

DEMARCHI, L.O. Composição, conhecimento e uso de plantas de campinarana por moradores da Reserva de Desenvolvimento Sustentável do Tupé - Amazônia Central. 2014. 82 f. Dissertação (Mestrado em Recursos Naturais) - Instituto Nacional de Pesquisas da Amazônia, Manaus, 2014.

EYSSARTIER, C. et al. Cultural transmission of traditional knowledge in two populations of North-western Patagonia. Journal of Ethnobiology and Ethnomedicine 4, 25, 2008.

FENTAHUN, M. T.; HAGER, H. Exploiting locally available resources for food and nutritional security enhancement: wild fruits diversity, potential and state of exploitation in the Amhara region of Ethiopia. Food Security, 1, 207-219, 2009.

FERRAZ, J.S.F. et al. Use-value and phytosociology of woody plants on the banks of the Riacho do Navio stream, Floresta, Pernambuco State, Brazil. Acta Botanica Brasilica, 20(1): 125-134, 2006.

FLORES A.S.; RODRIGUES R.S. Diversidade de Leguminosae em uma área de savana do estado de Roraima, Brasil. Acta Botanica Brasilica, 24: 175-183, 2010.

GALEANO, G. Forest use at the Pacific Coast of Chocó, Colômbia: a quantitative approach. Economic Botany, 54(3): 358-376, 2000.

GÓMEZ-BAGGETHUN, E.; V. REYES-GARCÍA. Reinterpreting change in traditional ecological knowledge. Human Ecology, 41 (4):643-647, 2013.

LADIO, A.; M. LOZADA. Human ecology, ethnobotany and traditional practices in a rural population of the Monte Region, Argentina: resilience and ecological knowledge. Journal of Arid Environments, 73: 222-227, 2009.

LINS NETO, E.M.F. et al. The knowledge and harvesting of Myracrondruon urundeuva Allemão by two rural communities in NE Brazil. Functional Ecosystems and Communities, 2: 66-71, 2008.

LUCENA, R.F.P. et al. Does the local availability of woody Caatinga plants (Northeastern Brazil) explain their use value? Economic Botany, 61(4): 347-361, 2007.

LUCENA, R.F.P. et al. The ecological apparency hypothesis and the importance of useful plants in rural communities from Northeastern Brazil: an assessment based on use value. Journal of Environmental Management, 96:106-115, 2012.

LUOGA, E. J. et al. Differential utilization and ethnobotany of trees in Kitulanghalo Forest Reserve and surrounding communal lands, eastern Tanzania. Economic Botany, 54(3): 328-343, 2000.

LYKKE, A. M. et al. Valuation of local use and dynamics of 56 woody species in the Sahel. Biodiversity and Conservation, 13: 1961-1990, 2004.

MELO, M.C.; BARBOSA, R.I. Árvores e arbustos das savanas de Roraima: guia de campo ilustrado. 1. ed. Boa Vista: PMBV/CONSEMMA, 2007.

OLIVEIRA, R. L. C. et al. Árvores úteis da comunidade Darora. 1. ed. Boa Vista: UERR Edições, 2017.

PHILLIPS, O.; GENTRY, A.H. The useful plants of Tambopata, Peru: II. Additional hypothesis testing in quantitative ethnobotany. Economic Botany, 47(1): 33-43, 1993.

QUINLAN, M.B.; QUINLAN, R.J. Modernization and medicinal plant knowledge in a Caribbean Horticultural Village. Medical Anthropology Quarterly, 21(2): 169-192, 2007.

RIBEIRO, J.E.L.S. et al. Flora da Reserva Ducke: guia de identificação das plantas vasculares de uma floresta de terra-firme na Amazônia Central. Manaus: INPA/DFID, 1999.

SOP, T.K. et al. Ethnobotanical knowledge and valuation of woody plant species: a comparative analysis of three ethnic groups from the sub-Sahel of Burkina Faso. Environment, Development and Sustainability, 14 : 627- 649, 2012.

SOUTO, T.; TICKTIN, T. Understanding interrelationships among predictors (age, gender, and origin) of local ecological knowledge. Economic Botany, 66: 149-164, 2012.

TAITA, P. Use of woody plants by locals in Mare aux Hippopotames Biosphere Reserve in western Burkina Faso. Biodiversity and Conservation, 12: 1205-1217, 2003.

TUNHOLI, V.P. et al. Availability and use of woody plants in an agrarian reform settlement in the Cerrado of the state of Goiás, Brazil. Acta Botanica Brasilica, 27(3): 604-612, 2013.

VIU, A.F.M. et al. Etnobotânica: uma questão de gênero? Revista Brasileira de Agroecologia, 5(1): 138-147, 2010

VOEKS, R.A. Are women reservoirs of traditional plant knowledge? Gender, ethnobotany and globalization in northeast Brazil. Singapore Journal of Tropical Geography, 28: 7-20, 2007.

VOEKS, R.A.; LEONY, A. Forgetting the forest: Assessing medicinal plant erosion in Eastern Brazil. Economic Botany, 58(sup.): 294-306, 2004.

WITTMANN, F. et al. Manual of trees from Central Amazonian varzea floodplains: taxonomy, ecology and use. 1. ed. Manaus: Editora INPA, 2010. 